\title{
RANDOM GENERALIZED FUNCTIONS OF LOCALLY FINITE ORDER
}

\author{
D. M. EAVES
}

1. It can be said that the purpose of replacing sample functions with sample generalized functions (sample Schwartzian distributions) is to obtain a theory that can deal with time-derivatives of sample functions that are merely continuous, or perhaps worse. (For an account of the basic theory of random generalized functions (RGF) see [1, Chapters III and IV].) As is well known, the restriction of a particular generalized (sample) function to the compact closure of an open set merely yields a derivative of a continuous function. (For the theory of generalized functions see [5].) A random generalized function is said to have locally finite order if, with probability one, its restriction to the compact closure of an open parameter set yields the derivative of a continuous sample function. In this paper it is first shown that all Gaussian RGF's have locally finite order (Theorem 4). We then show that local finiteness is equivalent with the condition that the Strong Law of Large Numbers (SLLN) hold (Theorem 9).

2. Let $D$ be the exact union of nuclear spaces $D K_{1}, D K_{2}, \cdots$. (See [1]. Gel'fand's exact union (tochnoe ob'edinenie) coincides with the strict inductive limit of [5].)

Let $D^{\prime}$ be the dual space of $D$. $P$ will denote an arbitrary probability measure on the Borel sets in $D^{\prime}$, continuous on cylinder sets (see [1]). $D K$ will denote an arbitrary nuclear subspace of $D$, and $D K^{\prime}$ its dual space. Let ||$_{1},||_{2}, \cdots$ be an increasing family of Hilbert norms in $D K$, generating its topology.

For each $n=1,2, \cdots$ let $D K^{n^{\prime}} \subset D K^{\prime}$ be that subspace of functionals that are continuous relative to the norm ||$_{n}$. Then $D K^{1 \prime}$ $\subset D K^{2 \prime} \subset \ldots$; in fact, $D K^{\prime}$ is the exact union of its closed subspaces $D K^{n \prime}$ (see $[4$, Exercise $22 \mathrm{C}(\mathrm{c})]$ ). It is easy to see that the restriction to $D K$ of functionals on $D$ is a continuous transformation from $D^{\prime}$ onto $D K^{\prime}$; hence the subspaces

$\mathscr{D}^{\prime} K^{n}=\left\{\Phi \in \mathscr{D}^{\prime}: \Phi\right.$ acts on $\mathscr{D} K$ continuously relative to $\left.\mid{ }_{n}\right\}$ of $D^{\prime}$ are closed.

$P$ will denote an arbitrary Gaussian probability measure in $D^{\prime}$,

Received by the editors August 23, 1967. 
continuous on the cylinder sets (see [1]). $E$ will denote its expected value, whether for complex-valued or $D^{\prime}$-valued functions.

Since $\left(D^{\prime}, P\right)$ is Gaussian, $P$ has a mean value $E \Phi \in D^{\prime}$, and $E|\Phi(\phi)|^{2}<\infty$ for each $\phi \in \mathscr{D}$; furthermore the map $(\phi, \theta) \rightarrow E \Phi(\phi) \Phi(\theta) *$ is continuous on $D \times D D$, and the map $\phi \rightarrow E \Phi(\phi)$ is an element of $D^{\prime}$. (Here the asterisk denotes complex conjugate.)

Let $\sigma D \subset L_{2}\left(D^{\prime}, P\right)$ be the $L_{2}\left(D^{\prime}, P\right)$-closed span of all the maps $\Phi \rightarrow \Phi(\phi)-E \Phi(\phi)$ on $D^{\prime}$, for $\phi \in \mathcal{D}$. By associating the map $\Phi \rightarrow \Phi(\phi)$ $-E \Phi(\phi)$ with each $\phi \in \mathscr{D}$ we obtain a continuous embedding of $D$ onto a dense subset of $\sigma D D$. Therefore, since $D$ possesses a countable dense subset, so also must $\sigma D$. But this ensures us of the existence of a complete orthonormal subset $X_{1}, X_{2}, \cdots$ for $\sigma D$. Being Gaussian, these random variables must be mutually independent. We can now obtain a representation for the restriction of $\left(D^{\prime}, P\right)$ to $D K$ :

THEOREM 1. There exist $n$ and $\chi_{1}, \chi_{2}, \cdots$ in $D K^{n^{\prime}}$ such that $\Phi(\phi)$ $=\sum_{k} \chi_{k}(\phi) X_{k}(\Phi)+E \Phi(\phi)$ for each $\phi \in D K$; the series converges in $L_{2}\left(D^{\prime}, P\right)$.

Proof. By the Abstract Kernel Theorem (see [1]), there exist $m$ and $p$ such that for all $\phi$ and $\Psi$ in $D K$,

$$
E(\Phi(\phi)-E \Phi(\phi))\left(\Phi(\Psi)^{*}-E \Phi(\Psi)\right)^{*}=\sum_{k=1}^{\infty} \lambda_{k}\left(\phi, \phi_{k}\right)_{p}\left(\Psi, \Psi_{k}\right)_{m},
$$

where $\left\{\phi_{k}\right\}$ and $\left\{\Psi_{k}\right\}$ are orthonormal bases of $D K^{p^{\prime}}$ and $D K^{m^{\prime}}$ respectively, $\lambda_{k} \geqq 0$ and $\sum_{k=1}^{\infty} \lambda_{k}<\infty$. It follows that the above inner product in $L_{2}\left(\mathscr{D}^{\prime}, P\right)$ is $\mid{ }_{p}$-continuous in $\phi$ and ||$_{m}$-continuous in $\Psi$. Define $\chi_{k}(\phi)=E(\Phi(\phi)-E \Phi(\phi)) X_{k}(\Phi)^{*}$. If $n \geqq \max \{m, p\}$ is so big that $E \Phi \in D^{\prime} K^{n}$, it follows that every $\chi_{k} \in D^{\prime} K_{n}$, and that $\Phi(\phi)$ has the promised representation.

Theorem 2. If $\left(D^{\prime}, P\right)$ is Gaussian, then $P\left(D^{\prime} K^{m}\right)=1$ for some $m$.

Proof. Let $n$ be as in Theorem 1 . It is easy to see that for every $m \geqq n, D^{\prime} K^{m}$ is a tail event for the sequence $X_{1}, X_{2}, \cdots$. By the Borel-Cantelli Lemma, $P\left(\mathscr{D}^{\prime} K^{m}\right)$ is either 0 or 1 . But $1=P\left(D^{\prime}\right)$ $=P\left(\bigcup_{m} \mathscr{D}^{\prime} K^{m}\right)$ implies $P\left(\mathscr{D}^{\prime} K^{m}\right)>0$ for some $m$; hence $P\left(\mathscr{D}^{\prime} K^{m}\right)=1$ for all sufficiently large $m$.

For the following lemma and theorem let the relatively compact regions $\Omega_{\nu}$ exhaust a subregion $\Omega$ of a real $N$-dimensional space, with $K_{\nu}=\bar{\Omega}_{\nu} \subset \Omega_{p+1}(\nu=1,2, \cdots)$; for an arbitrary compact neighborhood $K \subset \Omega$ let $D K$ be the space of infinitely differentiable complex-valued functions on $\Omega$ supported in $K$, with the (nuclear) topology generated by the norms $|0| \quad \mid 1,, \cdots$, where 


$$
|\phi|_{n}=\sum_{|k|=0}^{n} \int_{k}\left|\phi^{(k)}(x)\right|^{2} d x .
$$

(Here $|k|=\left|\left(k_{1}, \cdots, k_{N}\right)\right|=k_{1}+\cdots k_{N}$.)

Let the space $\mathcal{C} K$ have the same underlying set as in $D K$, but with the topology generated by the norms \|\|$_{0},\|\|_{1}, \cdots$, where

$$
\|\phi\|_{n}=\max _{|k|=0, \cdots, n} \sup _{x \in k}\left|\phi^{(k)}(x)\right| ;
$$

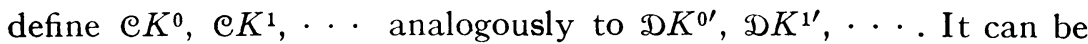
shown that for each $m$ there exists a $P$ for which $\mid p_{p}$-convergence implies \|\|$_{m}$-convergence, and also a $p$ for which \|\|$_{p \text {-con- }}$ vergence implies $|\quad|{ }_{m}$-convergence.

(See $[1$, Chapter $1, \S 3$, Theorem 7$]$ for the proof when $\Omega$ is the real line.) Hence the $\mathfrak{C} K$ topology coincides with the $\mathfrak{D} K$ topology.

These observations yield two important facts: First, the usual Schwartz topology in $\mathscr{D}$ (i.e., the exact union of the spaces $\mathfrak{e} K_{1}$, $\left.\mathcal{C} K_{2}, \cdots\right)$ makes $D$ the exact union of the nuclear spaces $D K_{1}, D K_{2}$; secondly, for each $m$ there exists a $p$ such that $D K^{m^{\prime}} \subset \mathbb{C} K^{p^{\prime}}$. (The $\mathcal{C} K^{p^{\prime}}$ are defined analogously to the $D K^{p^{\prime}}$.) We remark that in this context, the conditions on $P$ are precisely those that $\left(D^{\prime}, P\right)$ be a RGF on $\Omega$.

Lemma 3. If $\left(D^{\prime}, P\right)$ is Gaussian, then for some $m=m(K), P\{\Phi$ acts continuously on $\left.\mathrm{e} K^{m}\right\}=1$.

Proof. Take $\nu$ so big that $K_{\nu} \supset K$, let $n$ be so big that $P\left(D^{\prime} K_{\nu}^{n}\right)=1$, then take $m$ so large that $\mathcal{C} K^{m^{\prime}} \supset \mathbb{D} K^{n \prime}$.

Theorem 4. Let ( $\left.D^{\prime}, P\right)$ be Gaussian. Given any open $G \supset K, \Omega \supseteq G$, there exist $p=\left(p_{1}, \cdots, p_{N}\right)$ and a continuous $G$-supported function $g(\Phi, \cdot)$ for almost every $\Phi \in D^{\prime}$, such that $\Phi=g(\Phi, \cdot)^{(p)}$ on $K$. The exceptional $\Phi$-set is independent of $K$ and $G$. Hence $\left(D^{\prime}, P\right)$ has locally finite order.

Proof. For each $K=K_{\nu}(\nu=1,2, \cdots)$ let $m=m(\nu)$ be as in Lemma 3. Consider any $\Phi$ that acts continuously on every $\mathrm{e} K_{\nu}^{m(v)}$; let $\mu$ be so big that $K_{\mu} \supset K$. Since $\Phi$ acts continuously on $\mathrm{e} K^{m(\mu)}$, the conclusion follows by taking $p_{1}=p_{2}=\cdots=p_{N}=m(\mu)+2[\mathbf{5}$, Vol. 1, Theorem 21].

3. Let $\Phi^{1}, \Phi^{2}, \cdots$ be identically distributed independent random elements in a linear topological space $X$, distributed according to a measure $P$ continuous on the cylinder sets, for which a mean value 
$E \Phi$ exists in $X$. We shall say the Strong Law of Large Numbers (SLLN) holds in $X$ if, under these conditions, with probability one

$$
\frac{1}{n} \sum_{\nu=1}^{n} \Phi^{\nu} \stackrel{n}{\rightarrow} E \Phi \quad \text { in } X
$$

In this section we first show that if $X=D K^{\prime}$ is the dual of a nuclear space, then the SLLN holds if and only if $P\left(D K^{m \prime}\right)=1$ for some $m$. This will then be used to show that if $X=D^{\prime}$ is the space of generalized functions on a region $\Omega$, then the SLLN holds if and only if $(X, P)$ has locally finite order.

Lemma 5. Let $P$ be a probability measure in a separable locally convex space $X$; let $p$ be the gauge functional of any balanced, convex neighborhood in $X$. If $P$ has a mean value $E \Phi$, then $E p(\Phi)<\infty$.

Proof. Let $N$ be the null space of $p$, let $W$ be the $p$-completed quotient space of $X$ by $N$, normed by $p$; let $\pi$ be the quotient map of $X$ into $W$. Let $B$ be the smallest $\sigma$-ring of subsets of $W$ containing $\pi(X)$ as well as the Borel subsets of $W$. Define $R(B \cap \pi(X))$ $=P\left(\pi^{-1}(B)\right)$ for all $B \in B . R$ is then a probability measure on $B$, for which $\pi(\Phi)$ has the expected value $\pi(E \Phi)$ : for all continuous linear functionals $\phi$ on $W$,

$$
\phi(\pi(\Phi))=\int_{X} \phi(\pi(\Phi)) d P(\Phi)=\int_{W} \phi(w) d R(w) .
$$

Now in a separable Banach space (e.g., $W$ ), the mean value exists if and only if it exists as a Bochner integral. ([3, Theorem 3.7.2] proves this for functions taking only countably many values in the space; one then employs a countable dense subset to approximate the identity function $f(\Phi)=\Phi$ with functions assuming countably many values.) Furthermore the Bochner integral exists if and only if the norm has (finite) mean value [3, Theorem 3.7.4]. This finishes the proof, since

$$
E_{p}(\Phi)=E_{R} p(\pi(\Phi))<\infty .
$$

We now return to the notation preceding Theorem 2 .

THEOREM 6. The SLLN holds for $\left(D K^{\prime}, P\right)$ if and only if $P\left(D K^{m^{\prime}}\right)$ $=1$ for some $m$. (Here, $P$ is a probability measure in $D K^{\prime}$.)

Proof. Let $Q$ be the product measure in the sample sequence space $\prod_{k}\left(D K^{m^{\prime}}\right)^{k}$, whose projection onto each coordinate space $\left(D K^{m^{\prime}}\right)^{k}$ coincides with $P$. Write $\bar{\Phi}_{n}=(1 / n) \sum_{k=1}^{n} \Phi^{k}$ for each $n$. If the SLLN holds, then 


$$
\begin{aligned}
1 & =Q\left\{\bar{\Phi}_{n} \stackrel{n}{\rightarrow} E \Phi \text { in } D K^{\prime}\right\} \\
& \leqq Q\left\{\text { for some } m, \text { as } n \rightarrow \infty \text { eventually } \bar{\Phi}_{n} \in D K^{m^{\prime}}\right\} \\
& =Q\left\{\text { for some } m, \text { as } k \rightarrow \infty \text { eventually } \Phi^{k} \in D K^{m^{\prime}}\right\} \\
& =Q\left\{\text { for some } m, \Phi^{k} \in D K^{m^{\prime}} \text { for all } k\right\} .
\end{aligned}
$$

The first inequality simply expresses the meaning of convergence (in the exact union $D K^{\prime}$ of subspaces $D K^{m^{\prime}}$ ); the last three events named above all coincide. Thus for some $m$,

$$
0<Q \bigcap_{k}\left\{\Phi^{k} \in D K^{m^{\prime}}\right\}=\lim _{K \rightarrow \infty} Q \bigcap_{k=1}^{K}\left\{\Phi^{k} \in D K^{m^{\prime}}\right\}=\lim _{K \rightarrow \infty} P\left(D K^{m^{\prime}}\right)^{K} .
$$

For such $m P\left(D K^{m^{\prime}}\right)=1$ must hold.

Conversely, suppose there is an $m$ such that $P\left(D K^{m^{\prime}}\right)=1$. If necessary enlarge $m$ so that $E \Phi \in D K^{m^{\prime}}$. To show that the SLLN then holds for $P$ in $D K^{\prime}$ it is enough to show that it holds for the trace of $P$ in $D K^{m \prime}$. That this is satisfied follows from the SLLN for separable Banach spaces [2, Theorem 6.4.2], once it is seen that $E \Phi$ is the mean for the trace of $P$ in $D K^{m^{\prime}}$, as follows: Let $p$ be the gauge functional of a balanced, convex $D K^{\prime}$-neighborhood $U$ satisfying $U \cap D K^{m^{\prime}}=U_{m}^{0}$, where $U_{m}^{0}$ is the polar of the unit ||$_{m}$-ball. Then $p(\Phi)=|\Phi|_{-m}$ $=\sup \left|\Phi\left(U_{m}\right)\right|$ is the norm of $\Phi$ in the Hilbert space $D K^{m^{\prime}}$. Thus

$$
\int_{D_{K^{\prime \prime}}}|\Phi|_{-m} d P(\Phi)=\int_{\mathscr{D} K^{\prime}} p(\Phi) d P(\Phi)<\infty
$$

by Lemma 5 . This guarantees the existence of some mean $\Gamma$ for the trace of $P$ in $\mathscr{D} K^{m^{\prime}}$; i.e., $\Gamma(\phi)=\int_{D K^{m}} \Phi(\phi) d P(\phi)$ for all $\phi\left(|\phi|_{m}<\infty\right)$. But the functionals $\Phi \rightarrow \Phi(\phi)\left(|\phi|_{m}<\infty\right)$ form a dense subset of the dual space of $D K^{m^{\prime}}$; hence $(E \Phi)(\phi)=\int_{D K^{m^{\prime}}} \Phi(\phi) d P(\Phi)\left(|\phi|_{m}<\infty\right)$ implies $\Gamma=E \Phi$. Since the trace of $P$ in $D K^{m^{\prime}}$ has the mean $E \Phi$, the SLLN for separable Banach spaces implies $\bar{\Phi}_{n} \stackrel{n}{\rightarrow} E \Phi$ in $D K^{m^{\prime}}$, almost everywhere relative to the corresponding product measure in $\prod_{k}\left(D K^{m^{\prime}}\right)^{k}$. From this it can be shown that $\bar{\Phi}_{n} \stackrel{n}{\rightarrow} E \Phi$ in $D K^{m^{\prime}}$, and hence in $D K^{\prime}$, almost everywhere relative to $Q$.

Once again let $\left(D^{\prime}, P\right)$ be a RGF in a region $\Omega$, and for each compact closure $K \subset \Omega$ of an open set let $P_{K}$ be the projection of $P$ onto $D K^{\prime}$; i.e., $P_{K}(B)=P\{\Phi \mid K \in 囚\}$, where $\Phi \mid K$ is the restriction of $\Phi$ to $D K$. Let $Q$ and $Q_{K}$ respectively be the product measures in the sample sequence spaces $\prod_{i}\left(D^{\prime}\right)^{i}$ and $\prod_{i}\left(D K^{\prime}\right)^{i}$, corresponding to $P$ and $P_{K}$. To see what Theorem 6 means for random generalized functions, we need two lemmas: 
LEMma 7. If the SLLN holds for $\left(D^{\prime}, P\right)$ then it holds for every $\left(D K^{\prime}, P_{K}\right)$.

Proof. For each Borel set $B$ in $\prod_{i}\left(D K^{\prime}\right)^{i}$ let

$$
\widetilde{Q}_{K}(B)=Q\left(\left\{\left(\Phi_{1}\left|K, \Phi_{2}\right| K, \cdots\right) \in B\right\}\right) .
$$

$\left(Q_{K}\right.$ is a measure, since the map $\left(\Phi_{1}, \Phi_{2}, \cdots\right) \rightarrow\left(\left.\Phi_{1}\right|_{K},\left.\Phi_{2}\right|_{K}, \cdots\right)$ from $\prod_{i}\left(D^{\prime}\right)^{i}$ to $\prod_{i}\left(D K^{\prime}\right)^{i}$ is continuous relative to the product topologies.) Then $Q_{K}=\widetilde{Q}_{K}$, since they agree on the Borel rectangles.

If the SLLN holds in $\left(D^{\prime}, P\right)$ i.e., if

$$
Q\left(\left\{\bar{\Phi}_{n} \stackrel{n}{\rightarrow} E \Phi \text { in } \mathscr{D}^{\prime}\right\}\right)=1,
$$

then

$$
Q\left(\left\{\bar{\Phi}_{n}|K \stackrel{n}{\rightarrow}(E \Phi)| K \text { in } D K^{\prime}\right\}\right)=1,
$$

since the latter event contains the former. But this quantity by definition coincides with

$$
\widetilde{Q}_{K}\left(\left\{\bar{\Phi}_{n} \stackrel{n}{\rightarrow}(E \Phi) \mid K \text { in } \mathscr{D} K^{\prime}\right\}\right),
$$

and therefore with

$$
Q_{K}\left(\left\{\bar{\Phi}_{n} \stackrel{n}{\rightarrow}(E \Phi) \mid K \text { in } \mathscr{D} K^{\prime}\right\}\right) .
$$

To finish the proof, we merely note that $(E \Phi) \mid K$ is the expected value in $\left(D K^{\prime}, P_{K}\right)$, as follows: For all $\phi \in D K$,

$$
(E \Phi) \mid K(\phi)=(E \Phi)(\phi)=\int_{\Phi^{\prime}} \Phi(\phi) d P(\Phi)=\int_{\mathscr{D} K^{\prime}} \Phi(\phi) d P_{K}(\Phi) .
$$

\section{Lemma 8. Converse of Lemma 7.}

Proof. Let $K_{1}, K_{2}, \cdots$ be as in Lemma 3. By hypothesis, and in view of the remarks in the proof of Lemma 7 ,

$$
Q\left(\bigcap_{\nu}\left\{\bar{\Phi}_{n}\left|K_{\nu} \stackrel{n}{\rightarrow}(E \Phi)\right| K_{\nu} \text { in } D K_{\nu}^{\prime}\right\}\right)=1 .
$$

Now convergence in $D^{\prime}$ means uniform convergence on bounded subsets of $D$, and these are precisely the bounded subsets of the various $D K_{\nu}(\nu=1,2, \cdots)$. Furthermore convergence in each $D K_{\nu}^{\prime}$ means uniform convergence on bounded subsets of $D K_{v}$. This means the above event is smaller than $\left\{\bar{\Phi}_{n} \stackrel{n}{\rightarrow} E \Phi\right.$ in $\left.D^{\prime}\right\}$, finishing the proof. 
Theorem 9. The SLLN holds for a $R G F\left(D^{\prime}, P\right)$ if and only if it has locally finite order.

Proof. If the SLLN holds, then for each $\nu$, Lemma 7 and the "only if" part of Theorem 6 imply $P\left(D^{\prime} K_{\nu}^{m(\nu)}\right)=1$ for some $m(\nu)$. Given any $K$ with open $G, \Omega \supseteq G \supseteq K$, every $\Phi \in \bigcap_{\nu} D^{\prime} K_{\nu}^{m(\nu)}$ then has the form $\Phi=g(\Phi, \cdot)^{(p)}$ on $K$, where each $g(\Phi, \cdot)$ is a continuous $G$-supported function on $\Omega$. This is seen from the fact that $D^{\prime} K_{v}^{m(v)} \subset D^{\prime} K^{m(v)}$, followed by the proofs of Lemma 3 and Theorem 4 .

Conversely, if $\left(\mathscr{D}^{\prime}, P\right)$ has locally finite order then for each $K$ there exists $p=\left(p_{1}, \cdots, p_{N}\right)$ such that $P\left(\left\{\Phi \mid K\right.\right.$ agrees with $g(\Phi, \cdot)^{(p)}$ for some continuous function $g(\Phi, \cdot)\})=1$. But this event is smaller than $D^{\prime} K^{m}$, provided $m \geqq \max \left\{p_{1}, \cdots, p_{N}\right\}$. By Theorem $6,\left(D K^{\prime}, P_{K}\right)$ satisfies the SLLN. By Lemma $8,\left(D^{\prime}, P\right)$ satisfies the SLLN.

In conclusion, we would like to suggest that Fourier transform methods might be especially applicable in studying the structure of RGF's of locally finite order. These methods might be based on a global version of Theorem 4 . In order to extend these methods even further, it might be useful to obtain decomposition theorems representing RGF's linearly in terms of RGF's of locally finite order.

\section{REFERENCES}

1. I. M. Gel'fand and N. Ja. Vilenkin, Generalized functions. Vol. 4: Applications of harmonic analysis, Academic Press, New York, 1964.

2. U. Grenander, Probabilities on algebraic structures, Wiley, New York, 1963.

3. E. Hille and R. S. Phillips, Functional analysis and semi-groups, Amer. Math. Soc. Colloq. Publ., Vol. 31, Amer. Math. Soc., Providence, R. I., 1967.

4. J. L. Kelly, I. Namioka, et al., Linear topological spaces, Van Nostrand, Princeton, N. J., 1963.

5. L. Schwartz, Théorie des distributions, Vols. I, II, Hermann, Paris, 1957.

Simon Fraser UNIVERSITY 